\title{
L'épisode « San Junipero » de Black Mirror : les miroitements du post-humain
}

\section{Hélène Machinal}

\section{Q OpenEdition}

1 Journals

\section{Édition électronique}

URL : http://journals.openedition.org/tvseries/3169

DOI : 10.4000/tvseries.3169

ISSN : 2266-0909

\section{Éditeur}

GRIC - Groupe de recherche Identités et Cultures

\section{Référence électronique}

Hélène Machinal, «L'épisode « San Junipero » de Black Mirror: les miroitements du post-humain », TV/ Series [En ligne], 14 | 2018, mis en ligne le 31 décembre 2018, consulté le 30 avril 2019. URL : http:// journals.openedition.org/tvseries/3169; DOI : 10.4000/tvseries.3169

Ce document a été généré automatiquement le 30 avril 2019.

\section{(c) (i) (9)}

TV/Series est mis à disposition selon les termes de la licence Creative Commons Attribution - Pas d'Utilisation Commerciale - Pas de Modification 4.0 International. 


\title{
L'épisode « San Junipero » de Black Mirror : les miroitements du post- humain
}

\author{
Hélène Machinal
}

1 Le contexte de la troisième révolution industrielle a rapidement vu émerger une dynamique de réflexion sur la redéfinition potentielle de l'identité humaine entrânée par les biotechnologies (l'homme augmenté) et la société écranique (Serroy, Lipovetsky) ou hypermédiatique (Bertrand Gervais). Les séries TV d'anticipation sont devenues un genre majeur dans les années 1970. Depuis les années 2000, elles sont l'un des miroirs les plus révélateurs pour représenter les possibles évolutions de l'humain vers diverses formes d'hybridation et de mutations qui figurent les possibles ouverts par la perspective du post-humain. Black Mirror (C. Brooker, Channel4, Netflix, 2011- ) en est un exemple typique et d'autant plus intéressant que britannique, dans un univers médiatique dominé par les séries télévisées américaines. Cette série propose une esthétique relativement spécifique qui s'appuie, entre autres, sur des phénomènes de défamiliarisation suffisamment inhabituels pour qu'on s'y arrête. Elle est aussi emblématique d'un format qui s'inscrit à la fois dans le temps long de la sérialité et le temps court de l'épisode. Puisqu'elle s'incrit dans l'anthologie ${ }^{1}$, elle propose des épisodes dont le fonctionnement narratif et esthétique relève de la forme brève. L'épisode retenu pour cette analyse, «San Junipero ", est relativement unique dans sa façon d'introduire le novum et d'opérer la défamiliarisation cognitive. L'épisode se construit par ailleurs sur le mode de la rupture, motif typique du fantastique, que l'épisode décline à différents niveaux et qui a une portée méta-réeflexive.

2 Black Mirror est aussi une série paradoxale, qui tend aux téléspectatrices et téléspectateurs un miroir sans reflet, "a black mirror», dont «San Junipero » est une synecdoque. La série et l'épisode traitent en effet d'un "post» de l'humain dans une société hypermédiatique en s'attachant à la question de la mort et donc de la finitude définitoire de l'humain. L'épisode est en lien direct avec la globalité de la série puisque nous verrons que le miroir y est central, de l'intra-diégétique au méta-réflexif. Le miroir 
brisé renvoie ainsi à la possibilité d'une absence de reflet, ou d'un reflet qui renverrait à la fois à l'absence et à la présence, aux miroitements trompeurs de l'illusion et du simulacre associés au virtuel comme monde de l'après, de l'éternelle jeunesse et de la consommation d'un bonheur-artifice.

\section{Black Mirror et "San Junipero », les enjeux contemporains de la troisième révolution industrielle}

Black Mirror est à la fois à réinscrire dans l'essor contemporain des séries d'anticipation mais c'est aussi une série relativement spécifique, et ce peut-être d'abord parce qu'elle est britannique. Or, on retrouve dans les séries britanniques une propension à mettre à distance les modèles sériels américains, un aspect que les exemples de Misfits, Utopia ou Broadchurch ${ }^{2}$ peuvent illustrer. Black Mirror a pour fil rouge notre rapport aux médias, à la médiation et aux dispositifs technologiques dans un cadre bien spécifique, celui de nos sociétés hypermédiatiques. Comme le souligne Sébastien Lefait :

Plutôt qu'à la science ou à la technologie, Brooker s'intéresse par conséquent à l'humain. Son œuvre illustre les effets de cet « «écran global» dont Lipovestky et Serroy ont décrit l'avènement. Implicitement, Black Mirror exprime la même question que ces deux auteurs: "Quels sont les effets de cette prolifération d'écrans en matière de rapport au monde et aux autres, aux corps et aux sensations ${ }^{3}$ ?»

4 La série propose une réflexion sur la société écranique qui nous entoure, sur l'impact des dispositifs hypermédiatiques et sur l'augmentation de l'humain par la technologie, les trois aspects pouvant d'ailleurs se conjuguer. Black Mirror s'inscrit dans une réflexion sur les enjeux de la troisième révolution industrielle, dans laquelle on peut distinguer deux facettes: d'une part ce que l'on appelle parfois la fabrique du vivant ${ }^{4}$, donc les modifications (organiques ou pas) et augmentations apportées au corps humain; et, d'autre part, une société écranique et hypermédiatique dominée par la culture de l'écran. L'épisode « San Junipero » relève des deux. Il introduit en effet un univers virtuel dans lequel évoluent les personnages lorsqu'ils sont connectés. L'accès à cet espace virtuel repose également sur des dispositifs technologiques et sur une innovation qui ne relève encore que du possible: numériser un être humain ${ }^{5}$. Cet épisode nous invite à une réflexion sur le posthumain, un posthumain qui renvoie à la fois au corps et à l'esprit, puisque la technologie permet dans le monde diégétique de l'épisode de faire le choix d'une forme d'immortalité par téléchargement définitif de l'être en fin de vie dans un espace virtuel où il conserve tous ses souvenirs mais aussi, et ce n'est pas un détail anodin, le corps de sa jeunesse. L'homo numericus est-il dès lors l'avenir de l'homme (qui plus est un avenir éternel) ? Et sommes-nous promis à ce devenir ?

L'épisode est construit sur une approche paradoxale de la notion même d'augmentation ou de « mélioration » de l'humain et du vivant qui échapperait à sa condition de mortel et à sa finitude. En effet, l'accès définitif à cet espace virtuel associé à l'immortalité s'opère selon un processus qui s'apparente à l'euthanasie. La mort est centrale dans cet épisode qui nous raconte par ailleurs une histoire d'amour entre deux femmes, Kelly et Yorkie. Nous verrons que cette opposition classique entre Éros et Thanatos participe de la défamiliarisation cognitive à l'œuvre dans l'épisode. 


\section{Black Mirror, rupture et défamiliarisation}

6 Relevant de l'anthologie, Black Mirror adopte un format qui nous permet d'apparenter chaque épisode distinct à la forme brève, même s'ils constituent des entités à réinscrire dans un ensemble plus large mais qui relève davantage de la répétition par la variation que d'un continuum narratif. Chaque épisode va introduire une rupture, ce mode de construction d'un effet sur le récepteur constituant l'identité distinctive de la série. L'effet que porte en lui l'épisode « San Junipero » semble cependant unique dans la série. Le surgissement, voire le basculement, dans une perception du caractère alternatif de la réalité représentée dans la diégèse s'opère à la fois sur le mode de la rupture (en cours d'épisode) et du basculement final (extrêmement final puisqu'il s'agit du générique). Il y a donc là deux moments distincts et spécifiques qui sont centraux pour repérer l'hybridité générique à l'œuvre dans l'épisode.

7 Le phénomène de rupture opéré à mi-épisode renvoie à la séquence d'ouverture. Nous entrons dans cet épisode pour ainsi dire in medias res, avec une scène d'ouverture qui propose un gros plan soulignant le calme du mouvement des vagues sur le sable, avant un fondu enchaîné sur les lumières d'une ville. La bande son est importante car la chanson mielleuse à souhait «Heaven is a Place on Earth" annonce la facture esthétique de l'épisode et son inscription générique dans la comédie romantique. Elle sert de marqueur temporel identifiant l'année 1987, tout comme l'affiche de film The Lost Boys (Génération perdue en français), même si les spectatrices et spectateurs ne savent pas encore que la chanson comme l'affiche pointent déjà plus qu'une simple date. La chanson est reprise pendant le générique de fin, ce qui inscrit aussi l'épisode dans la cyclicité, mais introduit une discordance car le paradis du générique de fin relève de l'artifice et du simulacre virtuel, un endroit bel et bien situé sur terre mais pas au sens où l'entend la chanson. De même, l'intertexte « the Lost Boys » semble s'avérer polysémique ${ }^{6}$. Le plan suivant nous introduit dans ce lieu dont nous ne savons pas encore qu'il est censé être un paradis sur terre par un travelling oblique qui place aussi l'œil de la caméra dans une dynamique descendante et nous fait découvrir la ville en plongée. L'endroit semble animé par une joyeuse vie nocturne, marquée par la jeunesse (ou le jeunisme?) des passants, tous pris dans le tourbillon de la fête. On note d'emblée l'omniprésence des cadres, des affiches et des écrans, de télévision en particulier. Le lieu et les tenues vestimentaires indiquent par ailleurs à nouveau un cadre diégétique passé, l'année 1987 aux États-Unis. Malgré le décalage temporel, le décor se veut donc mimétique et familier. Les dialogues entre les personnages introduisent cependant immédiatement une distinction entre l'ici et l'ailleurs : ici, c'est donc à San Junipero, un lieu synonyme de liberté et de plaisir ${ }^{7}$. C'est dans ce décor que Yorkie rencontre Kelly pour la première fois.

8 Cette ouverture se termine par un gros plan sur le reflet de la lune dans une flaque d'eau, le reflet de l'astre étant déformé par les gouttes de pluie qui tombent. S'ensuit un passage au noir accompagné d'un effet sonore de type larsen. Puis l'intertitre « One week later » s'affiche à l'écran (13'15-13'23). La séquence suivante file la rencontre amoureuse entre les deux jeunes filles et se termine par un gros plan sur le radio réveil au moment où il passe de 23 h59 à minuit (24'16), tandis que l'écran repasse au noir avec le même son de larsen que lors du premier raccord de ce type. On retrouve également la mention «One week later ». Ces écrans noirs sur lesquels apparaît le passage du temps (et qui rappellent les techniques du cinéma muet pour indiquer une ellipse temporelle) vont dès lors se 
succéder à un rythme qui s'accélère. Yorkie est à la recherche de Kelly ; un ex petit-ami à elle lui conseille « d'essayer une autre époque, les années 80 et 90, ou 2002 aussi » (27'13). Nous avons ainsi trois écrans noirs successifs en l'espace de deux minutes (27'32, 28'10, 29 '00), qui correspondent chacun à l'introduction d'une temporalité diégétique différente (les années 80 , puis 90 , puis 2000). À ce stade, les récepteurs extradiégétiques que nous sommes peuvent penser que cette ville est une sorte de parc à thème à la Westworld qui s'inscrirait dans une époque différente chaque semaine. Chaque époque est singularisée en premier lieu par les images que diffuse la télévision, par les affiches de films mais aussi par les tenues vestimentaires des protagonistes et, enfin, par les décors et les musiques des boîtes de nuit ou les jeux qui y sont proposés. La population reste toujours exclusivement composée de jeunes, détail qui peut commencer à poser question.

La rupture narrative et fictionnelle va s'opérer en deux temps successifs après que Kelly a rejeté Yorkie ${ }^{8}$ qui semble désormais éperdument amoureuse d'elle. Une première scène montre une Kelly visiblement furieuse face à un miroir. Le regard de Kelly avant son geste est important car on a là un regard caméra central pour la dimension méta-réflexive (cf.

Fig. 1).

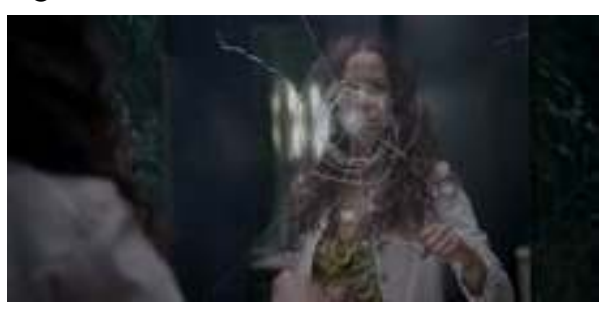

Fig. 1 : Kelly face au miroir

10 Kelly lance son poing fermé dans le miroir qui, comme de bien entendu, se brise. La rupture s'opère à ce moment précis (31'27) puisque l'image suivante dévoile Kelly regardant son poing et constatant qu'elle n'a aucune blessure ni même trace sur la main, puis la caméra remonte de la main vers... un miroir intact. Les spectatrices et les spectateurs constatent ainsi pour la première fois que la réalité qu'ils ont jusqu'alors considérée comme une réalité mimétique ne l'est sans doute pas, et que des phénomènes inexplicables s'y produisent. Ces doutes sont bien entendu étayés par le regard caméra qui contribue à souligner l'importance du plan séquence, et le fait que l'impact du poing comme le motif du miroir brisé sont emblématiques à la fois de l'épisode, mais aussi du générique de l'ensemble des épisodes de la série.

Kelly rejoint ensuite Yorkie et assises sur le toit d'un bâtiment, elles contemplent les groupes de jeunes à leurs pieds. On assiste alors à l'échange suivant, qui confirme nos soupçons sur la nature spécifique de ce cadre diégétique :

YORKIE. Combien d'entre eux sont morts? En pourcentage, t'as une idée?

KELLY. Tu veux dire pour de bon ? $80 \%$, peut-être 85 (32'36-32'46) ${ }^{9}$

La succession de ces deux scènes est centrale car elle scelle la rupture entre le monde diégétique et le réel des récepteurs. La réalité représentée n'est plus mimétique de la nôtre et on penche même à ce stade vers le fantastique puisque les lois de la physique sont remises en cause, et que les protagonistes que nous avons suivis depuis 30 minutes d'épisode sont déclarés morts « à plein temps » (« full-timers » en anglais) pour 80 à $85 \%$ d'entre eux. Ainsi la mort et l'image (ou le reflet) s'imposent (par «dissonance cognitive ») comme les motifs centraux qui vont dorénavant dominer toute la seconde partie de l'épisode. On apprendra ainsi que chacune des deux jeunes filles a été marquée 
par une (ou plusieurs) morts traumatiques (dans le cas de Yorkie, on pourrait presque dire la sienne propre). On va aussi découvrir que le monde de San Junipero n'est qu'un reflet, une image, un monde virtuel que les personnes en fin de vie peuvent choisir de rejoindre en optant pour l'euthanasie.

La première mort traumatique est rapportée par Kelly, qui explique que son mari est décédé deux années auparavant et qu'il n'a pas voulu «sauter le pas» ("pass over », expression plus intéressante en anglais), qu'il a refusé l'opportunité qui lui était offerte de "rester à San Junipero » parce qu'il "n'y croyait pas » (« did not believe in [it]») (36'13-36'35). La rhétorique de la croyance, de l'illusion, du simulacre est ainsi introduite et se confirme lorsque la conversation entre les deux jeunes femmes s'oriente vers une rencontre " pour de vrai » (« really», 37'16). Si les récepteurs avaient encore un doute, ils n'en auraient plus désormais : ce « pour de vrai » implique clairement que les images que nous avons suivies de semaine en semaine - que l'ensemble de ce monde fictif s'inscrivent dans le "pour de faux", et donc dans le virtuel. Ce que je qualifierais volontiers de "moment fantastique " (car la séquence suivante va nous faire basculer dans l'anticipation) ne dure donc pas et nous découvrons que nous avons été pris au piège d'un double décalage fictionnel. Le "vrai » correspond en fait à une réalité diégétique où les deux personnages sont en fin de vie, un monde où il est possible de passer quelques heures par semaine dans l'univers virtuel avant de devoir faire le choix de l'intégrer définitivement ou pas. Ce que nous avions pris pour une réalité (fictive certes mais mimétique) pendant 30 minutes d'épisode est une fiction, plus précisément, un univers virtuel, dans une mise en abyme de la fiction (cf. Fig. 2a et $2 b$ ).
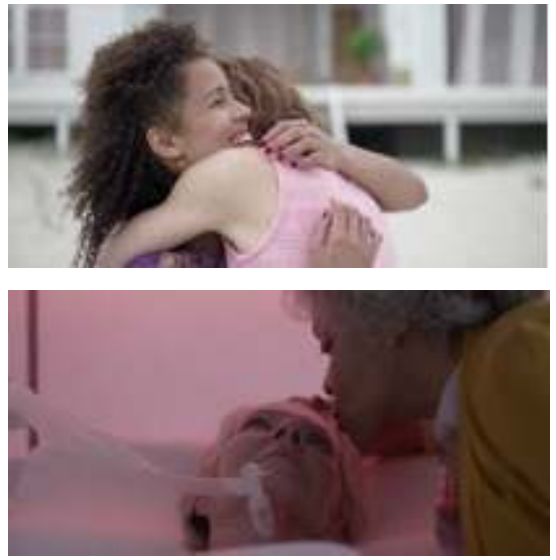

Fig. 2a et 2b : Le moment de bascule (du mimétique au virtuel)

La bascule qui s'opère à la moitié de l'épisode permet aussi de comprendre le rapport au temps que les personnages ont déjà amplement souligné, et ce dès le début de l'épisode. On note de multiples remarques sur le "peu de temps qu'il reste", qui s'ajoutent aux écrans noirs portant la mention "One week later », ou les cadrans de réveil affichant une heure qui se rapproche de ce qui paraît être une borne temporelle assez convenue depuis Cendrillon et/ou le roman gothique, c'est-à-dire l'heure de minuit. La temporalité est donc aussi associée au temps de la vie et à la mort comme caractéristique de notre condition de mortels ${ }^{10}$. L'univers fictif de San Junipero, qui semblait déborder de vie et de jeunesse ${ }^{11}$, est un royaume des morts "à plein temps » et la réalité diégétique non virtuelle se résume à deux espaces, les centres médicalisés où vivent respectivement les deux femmes en fin de vie. La seconde moitié de l'épisode va maintenir en parallèle ces deux espaces- 
temps, l'un mimétique du réel, l'autre totalement virtuel, l'un caractérisé par une vie simulacre, l'autre marqué du sceau de la mort et du trauma.

\section{L'oxymore du miroir noir, une série paradoxe}

15 «San Junipero» repose donc sur l'opposition et la tension entre les contraires, des sphères antithétiques l'une de l'autre. On a évoqué Eros et Thanatos, la réalité et le simulacre, le vrai et le faux, mais l'épisode traite aussi de l'identité sexuelle par une esthétique spécifique qui pose l'homosexualité en altérité sexuelle et inclut le spectateur ou la spectatrice dans ce regard clivé. Nous allons aussi voir qu'altérité sexuelle et altérité humaine, ou alternative à l'humain, sont par ailleurs mis en résonance.

L'épisode introduit la notion d'altérité lors du premier plan-séquence où l'on voit Yorkie marcher à contre-courant du flot de la jeunesse insouciante qui peuple la ville ${ }^{12}$. L'homosexualité va ensuite devenir centrale lors de la première séquence dans la boîte de nuit. Le malaise de Yorkie lorsqu'elle danse avec Kelly est d'abord souligné par une esthétique spécifique qui s'appuie sur le gros plan, des plans extrêmement rapprochés et décadrés et un regard caméra systématique. Cette esthétique donne au récepteur l'impression que c'est lui ou elle qui subit le malaise ressenti par Yorkie. La caméra subjective et le regard caméra opèrent en effet à plein car ils placent les spectateurs à la fois en position de voyeurs, à l'origine de ce regard rapproché et déformant, mais aussi en récepteurs du malaise. Dès qu'elle a trouvé refuge dans la ruelle, Yorkie énonce sa peur de l'homophobie (9'58). C'est ensuite son histoire, et en particulier le rejet de ce que sa famille considère comme son altérité, qui sont évoqués, avant que, suite aux avances que lui fait Kelly, elle annonce qu'elle doit se marier prochainement.

$\mathrm{Au}$ cours de cette première partie de l'épisode, les spectateurs attentifs notent aussi chez Yorkie une sensibilité aux voitures, et plus spécifiquement aux accidents. Elle refuse par exemple de jouer à un jeu vidéo où les voitures renversent allègrement les passants et percutent tout ce qui se trouve sur leur route. Elle semble très profondément affectée lorsqu'elle fait une sortie de route avec Kelly, puis lorsque Kelly a un accident qui est présenté comme une tentative de suicide (virtuelle, certes ${ }^{13}$ ). Ces détails vont trouver sens en s'ancrant dans un contexte traumatique expliqué par Greg, l'aide-soignant qui a proposé d'épouser Yorkie. Ces explications sont données très tard (41'00-43'). Quarante années se sont écoulées depuis le coming out de Yorkie à l'âge de vingt-et-un ans et son destin funeste : sa famille la rejette, elle part en voiture et a un très grave accident (qui était peut-être une tentative de suicide). Elle restera paraplégique pendant quarante années. L'épisode met donc en perspective le rejet d'une identité sexuelle assimilée à l'altérité, quelle que soit l'époque, mais il ne se contente pas d'aborder le thème de l'homosexualité et sa stigmatisation en altérité sexuelle. Il propose une réflexion plus subtile qui s'inscrit dans la mise à distance et la parodie du genre de la comédie romantique et de ses clichés au cinéma. Le recours à une esthétique du cliché, qui est dès lors révélé comme simulacre, va aussi permettre de jalonner le passage de l'altérité sexuelle à l'altérité (post)humaine.

Dès la première scène de déclaration d'amour entre Yorkie et Kelly, ces clichés sont omniprésents. Les deux jeunes femmes sont isolées sur le toit d'un bâtiment qui surplombe le reste de la ville: leur histoire naissante les singularise déjà dans l'espace diégétique. Leur première relation sexuelle se passe dans une maison isolée en bord de 
mer, et on observe lors de cette séquence la traditionnelle ellipse narrative héritée du cinéma classique qui souligne que l'acte a eu lieu. Ici ce n'est pas le gros plan sur les flammes du feu de cheminée qui concrétise l'ellipse mais le gros plan sur les vagues léchant le sable (20'41). On pourrait même aller jusqu'à se demander si le cliché n'est pas ici lui-même genré, et ce de façon relativement transparente, tant l'opposition masculin/ féminin associé au feu et à la mer relève elle aussi du cliché. De même, les deux scènes de retrouvailles des épouses sont marquées du même sceau. Lorsque Yorkie « renaît » dans le monde virtuel de San Junipero, les deux jeunes femmes se retrouvent en robes blanches de mariées, Yorkie jette son voile à la mer, et elles s'éloignent dans la traditionnelle voiture rouge traînant des boites de conserves vides derrière elle. On retrouve exactement la même parodie des images convenues de la comédie romantique à la toute fin de l'épisode, lorsque Kelly rejoint définitivement Yorkie. On assiste en effet alors à la clôture type de l'histoire d'amour " qui finit bien » : le couple en voiture de sport rouge (comme il se doit), s'éloigne à l'horizon, dans un paysage de dune et de bord de mer sur fond de coucher de soleil. Le couple s'embrasse (toujours comme il se doit), et la bandeson rappelle « Heaven is a Place on Earth» (cf. figures 3a et 3b).
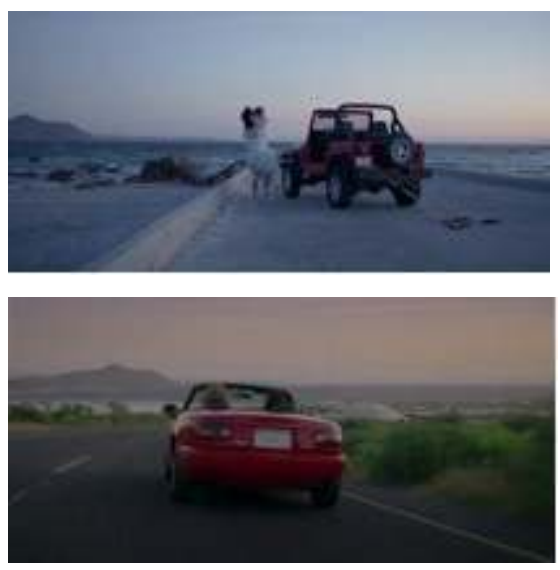

Fig. 3a et 3b : Plans romantiques et clichés

19 On pourrait ainsi penser que cette histoire a un dénouement heureux, et d'une certaine façon, c'est le cas, si l'on se cantonne au niveau intra-diégétique ${ }^{14}$. Mais on peut lire autrement ces scènes typiques des comédies romantiques, et de leur esthétique du cliché. En effet, les trois scènes - la première sur le toit de la boîte de nuit (32'), la seconde lorsque Yorkie « renaît » dans la réalité virtuelle (47'23), la dernière pendant le générique - sont également associées à la mort et au trauma. La comédie romantique et son esthétique artificielle sont donc en quelque sorte minées par cette résurgence compulsive du trauma lié à la perte. La mort de la fille de Kelly, celle de l'époux avec qui elle a partagé quarante années de vie, et l'impossibilité de les "retrouver ", même virtuellement dans ce monde fictif et simulacre qu'est San Junipero, confèrent à ces événements une épaisseur, une texture et une authenticité qui contraste avec la facticité de cette «vie éternelle ${ }^{15}$ » qui est, selon les termes de Kelly, "vendue » pour que les résidents permanents de ce monde-simulacre puissent «se sentir vivants ${ }^{16}$ ». Parallèlement aux images clichés des épouses, donc, l'épisode distille un retour constant à la réalité de la condition de mortelles, et d'êtres inscrits dans la finitude et la perte. L'abondance et la récurrence des images-clichés exacerbent le soubassement traumatique lié à la mort et à l'absence qu'elles ne parviendront pas à compenser. Il y a là une tension particulièrement sensible pour les spectateurs qui assistent par ailleurs aux deux scènes d'euthanasie, qui 
sont, au contraire, filmées sans recours à aucun type de cliché, avec un esthétique beaucoup plus naturaliste ${ }^{17}$. Scènes poignantes s'il en est, la mort des deux personnages principaux est perçue comme bien plus «réelle ${ }^{18}$ » et authentique que les clichés de la résurrection en avatars éternellement jeunes, heureux et insouciants. La concomitance de ces deux scènes d'euthanasie - que la mise en parallèle rend encore plus poignantes - avec ces scènes de comédie romantique clairement ancrées dans l'artifice de l'image et du simulacre, produit un phénomène de défamiliarisation dont la cognition est l'exclusivité du récepteur. La dimension méta-réflexive opère ici à plein. Les spectatrices et spectateurs ne peuvent, comme le mari de Kelly d'ailleurs, "croire » à ce monde idéal, et ne s'immergeront pas dans ce monde possible, pour reprendre les termes de Ryan et Besson ${ }^{19}$, car bien que l'épisode les ait immergés d'emblée dans un monde fictif donné pour mimétique pendant trente minutes d'épisode, le processus de prise de conscience de la facticité dudit monde empêche définitivement de suspendre son incrédulité ; l'immersion est par conséquent refusée. Le monde possible est ravalé au rang d'entité fictive, donné voire dénoncé comme fiction factice.

Cette porte ouverte vers un monde possible se referme d'ailleurs définitivement lors du générique de fin de l'épisode qui opère un basculement dans le posthumain et une rupture définitive - tout en inscrivant l'épisode dans le cyclicité. On l'a vu, dans ce même générique final, une série de plans sont alternés entre images-clichés du couple disparaissant dans l'horizon baigné d'un coucher de soleil et archivage des deux êtres numérisés dans cet univers virtuel. Nous entrons ainsi de plain-pied dans le posthumain, par une représentation directe, exclusivement réservée au récepteur. C'est lors du générique final - logiquement post-diégèse, en tout cas dans le cadre du générique de fin qui nous fait déjà franchir le pas de retour vers le réel - que cette révélation est effectuée. La série en profite pour cultiver à nouveau le paradoxe et les oppositions, ici entre diégèse et post-diégèse, mais aussi entre humain et posthumain, puisque les sentiments, l'amour, les affects et leur centralité pour l'être humain sont réduits (littéralement et par synecdoque) à des pastilles insérées par un robot dans une sorte de gigantesque matrice, opération que le spectateur est le seul à découvrir à la toute fin du générique (cf. Fig. 4a et $4 \mathrm{~b})$.
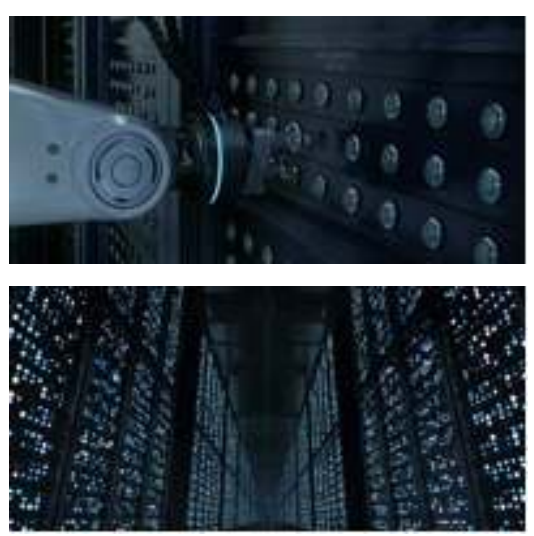

Fig. $4 a$ et $4 b$ : Pastilles informatiques et matrice posthumaine

On retrouve ici le même motif que dans Matrix ${ }^{20}$ ou Helix ${ }^{21}$, à ceci près que dans le premier, le héros intradiégétique, Néo, voit cette "fabrique du vivant», tandis que dans Helix ou "San Junipero ", seul le récepteur a ce privilège. Les deux jeunes femmes nageant dans le bonheur de leurs retrouvailles éternelles sont en réalité deux pastilles insérées dans une 
gigantesque matrice qui en contient une infinité. Ces images finales évoquent donc des images transfuges d'autres films et séries qui traitent de la production ou du traitement industriels d'êtres vivants - leur origine remonte à Brave New World ${ }^{22}$ et on les retrouve, tout près de nous, dans la première scène de Blade Runner $2049^{23}$. Le récepteur est donc le seul à subir la défamiliarisation cognitive opérée par la seconde moitié de l'épisode et condensée dans le générique final par l'alternance du générique de fin avec deux types de scènes aux esthétiques tellement opposées qu'elles produisent un frisson similaire à celui produit par la craie qui crisse sur le tableau noir. Esthétique, au sens de science de la connaissance sensible, saisie immédiate et intuitive du spectateur, qui place le récepteur en porte-à-faux concrétisé aussi dans le son de larsen qui accompagne les passages au noir avec leur mention "One week later », ou dans l'effet de « rupture » lorsque le miroir noir du générique de début se brise. En effet, les images-matrices sont synonymes de déshumanisation totale, d'une réduction de l'humain au plus petit dénominateur commun technologique. Finalement, les scènes alternées se jouent entre d'une part clichés de la comédie romantique et du « happy end » et, d'autre part, esthétique du touttechnologique qui relève du posthumain, monde dans lequel l'humain (en tout cas sa présence physique réelle et non virtuelle) a totalement disparu au profit du robotique, de la reproduction mécanique, et de l'archivage.

\section{Conclusion : Les miroitements du posthumain}

Black Mirror est une série qui traite de notre rapport à la surface, aux apparences, et du caractère illusoire de certains types de rapport à l'image. Il y a toujours deux faces au miroir ou deux niveaux de profondeur: sa surface qui se conjugue avec apparence et reflet, mais aussi sa profondeur, le célèbre "in a glass darkly » de LeFanu, qui introduit un espace certes plus trouble et sombre, mais porteur de mises en perspective. L'écran donne à voir les miroitements d'un univers posthumain : l'épisode "San Junipero " peut être analysé comme un miroir-leurre qui nous est tendu pour mieux exposer ensuite le factice d'une immortalité virtuelle en nous révélant l'envers du décor forme de «bris » symbolique de la surface, au-delà du geste de Kelly dans l'épisode ou du miroir brisé qui clôt chaque diffusion du générique de la série.

Ainsi, cet épisode, en apparence sympathique histoire d'amour au happy end convenu, va acquérir une profondeur sombre et glaçante. C'est d'abord la forme brève qui produit un effet maximal : un épisode se regarde selon les règles posées par Poe dans Philosophy of Composition, en une seule fois (" one sitting»), et il tend alors vers l'effet spécifique que j'ai isolé avec la rupture à mi-parcours et la chute du générique de fin. Le récepteur subit alors un processus de "dés-immersion » forcé, et se trouve extrait du monde fictif; l'immersion et le «désir de créance ${ }^{24}$ » dont parlent Besson et Ryan lui sont niés ${ }^{25}$. Ensuite, la dynamique de tension constante, en particulier entre identité et altérité, entre vie et mort et entre humain et posthumain, place les spectateurs dans cette même tension entre reconnaissance et défamiliarisation. L'épisode présente certes une histoire d'amour mais problématise notre rapport à la mort et nous place face à notre finitude de mortels. D'une certaine façon, Kelly souligne déjà ce décalage au niveau sémantique lorsqu'elle évoque les euphémismes utilisés ("That's what they say ») pour décrire cette immersion dans une réalité virtuelle. On parle en effet de "programme médical » et de thérapie par « immersion nostalgique [dans] l'univers de [la] mémoire » (42'46). Elle ajoute ensuite, 
cette fois à propos de la mort, "Just call it dying» (43'38), refusant ainsi clairement l'euphémisation de Greg ( to be scheduled to pass »).

«San Junipero » traite donc aussi du caractère illusoire de l'image, même lorsque la technologie semble pallier la perception du simulacre. En effet, lorsque Yorkie meurt et se retrouve dans le virtuel, elle souligne à quel point tout lui semble «tellement réel ${ }^{26}$ ». Là encore, les images pointent un ancrage dans la corporéité, dans la matérialité du toucher et dans les sens en général, mais la séquence (47’04-48'43) se termine par un gros plan sur les verres des lunettes abandonnées par Yorkie : la symbolique est claire, ce que nous voyons est sujet à caution, ce qui est donné à voir peut relever du simulacre, l'écran peut occulter le réel, littéralement «faire écran ». Au miroir qui ne se brise pas dans la diégèse parce que les personnages veulent croire à ce simulacre de fiction, il faut peutêtre substituer le miroir noir qui, lui, se brise dans le générique.

\section{NOTES}

1. Là encore, le phénomène remonte au milieu du $\mathrm{xx}^{\mathrm{e}}$ siècle avec, par exemple, The Twilight Zone.

2. Misfits (Howard Overman, E4, 2009-2013); Utopia (Dennis Kelly, Channel 4, 2013-2014); Broadchurch (Chris Chibnall, ITV, 2013-2017).

3. Sebastien Lefait, " 'It's not a technological problem we have, it's a human one' Black Mirror, ou la dystopie intégrée », in Elaine Després et Hélène Machinal (éd.), L'imaginaire en séries I, Otrante, n ${ }^{\circ} 42$, Paris, Kimé, 2017, p. 127-144.

4. Voir Yves Michaud, Humain, inhumain, trop humain, Réflexions sur les biotechnologies, la vie et la conservation de soi à partir de l'œuvre de Peter Sloterdijk, Paris, Climats, 2002. Voir aussi les ouvrages de Michel Serres, Hominescence, Paris, Le Pommier, 2001, et de Dominique Lecourt, Humain, Posthumain, Paris, PUF, 2003.

5. Ce motif n'est cependant pas nouveau ni dans les séries ni au cinéma. Par exemple, Caprica, prequel de Battlestar Galactica, propose le personnage de Zoe qui est triple, puisqu'elle se décline en une version organique et humaine, une version avatar virtuel, et une version robot. Voir Hélène Machinal, «Corps technologiques et environnement connectés : "The dynamic, fluid nature of reality' ou le corps face à l'écran ", TV/Series 11, Philosopher avec Battlestar Galactica, $\mathrm{n}^{\circ}$ 11, 2017, https://journals.openedition.org/tvseries/1341.

6. Le titre peut renvoyer aux êtres qui peuplent San Junipero dans leur ensemble (en tant que génération, factice puisque la notion de génération ne signifie plus grand-chose dans ce lieu), mais peut-être aussi aux garçons éconduits par Kelly comme par Yorkie. Par ailleurs, le film renvoie à Peter Pan et donc au syndrome du même nom (merci à David Roche pour cette remarque).

7. Voir 11'18, «San Junipero est le meilleur endroit pour faire la fête », « les gens sont vachement moins coincés quand ils viennent ici».

8. « In the time I've been here, I said I wouldn't - I don't know, do feelings. You freaked me out. I don't want to like anyone. So you've been just totally... fucking incovenient. » (33'40)

9. « How many of them are dead? Like, what percentage? / «As in full-timers ?... 80, 85 » (32'40).

10. N'oublions pas qu'en outre, Kelly annonce qu'elle est atteinte d'un cancer et qu'on "lui a donné trois mois » $\left(35^{\prime} 22\right)$. Le caractère éphémère de la vie avait déjà été évoqué lors de la 
première scène lorsque Kelly prétend, pour se débarrasser d'un soupirant, que Yorkie est atteinte d'une maladie incurable. Les deux personnages principaux sont donc en réalité en fin de vie, et le temps octroyé à San Junipero renvoie aussi au temps de vie qui leur reste.

11. On peut rappeler la symbolique associée à l'arbre (le genévrier ou «junipero ») qui est la fertilité.

12. Cela dit, Yorkie marche de la gauche vers la droite de l'écran, tandis que les passants s'orientent vers la gauche, sens dont la symbolique pointe la régression et le passé davantage que l'évolution et le futur.

13. Là encore, il y aurait beaucoup à dire sur cet accident et l'apparente mort de Kelly (qui reproduit aussi l'accident de Yorkie quarante ans plus tôt), qui a lieu exactement avant l'heure fatidique de minuit et la sortie du monde virtuel. Cette séquence contribue à renforcer les doutes sur la possibilité de mourir dans le monde virtuel.

14. Il est d'ailleurs amusant de constater que les réactions de récepteurs sur le net pointent parfois ce contraste, en particulier par rapport aux autres épisodes de la saison 3. "San Junipero » a pu être vu comme plus rassurant et moins déstabilisant, ce qui n'est absolument pas le cas.

15. 53'34

16. $53^{\prime} 56$

17. On pourrait aussi avancer que le montage alterné met en regard ces deux esthétiques.

18. Le terme est ici employé à dessein et permet de rappeler que c'est précisément ce sur quoi Yorkie insiste lorsqu'elle est définitivement à San Junipero : « It feels so real » (49'30)

19. Laurent Bazin, Anne Besson, Nathalie Prince (éd.), Mondes fictionnels, mondes numériques, mondes possibles, Rennes, PUR, 2016. Voir en particulier l'introduction d'Anne Besson et l'article de Marie-Laure Ryan.

20. Matrix (réal. A. et L. Wachowski, 1999).

21. Helix (Cameron Porsandeh, Syfy, 2014-2015).

22. Voir Hélène Machinal, «Brave New World : image-matrice et méta-iconicité, à partir d'Helix et Matrix », Patrick Bergeron (éd.), Otrante, n40, Paris, Kimé, 2016, p. 27-40.

23. Blade Runner 2049 (réal. Denis Villeneuve, 2017).

24. "[...] plutôt que «suspension de l'incrédulité », il y aurait là "création active de créance » [ « we actively create belief »] chez le récepteur, qui prend part à son immersion en s'efforçant de renforcer plutôt que de mettre en question la réalité de l'expérience » (Bazin, Besson et al., Mondes fictifs, p. 17).

25. Poe évoque aussi une cyclicité de la forme brève, que l'on retrouve dans « San Junipero ». 26. «So real» $\left(49^{\prime} 30\right)$.

\section{RÉSUMÉS}

Dans le contexte de la troisième révolution industrielle et de la redéfinition potentielle de l'identité humaine qu'entraînent les biotechnologies (l'homme augmenté) et la société écranique (Serroy, Lipovetsky) ou hypermédiatique (Bertrand Gervais), les séries TV d'anticipation sont l'un des miroirs le plus prégnants pour figurer les possibles évolutions de l'humain vers diverses formes d'hybridation et de mutation qui déclinent les possibles du posthumain. La série étant un format qui s'inscrit à la fois dans le temps long de la sérialité et le temps court de l'épisode, on 
analysera ici les modes de fonctionnement narratifs et esthétiques de la forme brève que prend l'épisode S03E04 de la Black Mirror (C. Brooker, Channel 4, Netflix, 2011- en production) intitulé «San Junipero». La série a pour particularité d'opérer une défamiliarisation du récepteur qui passe souvent par l'introduction d'un novum en cours d'épisode : l'épisode « San Junipero » met en scène la possibilité d'une vie éternelle virtuelle. Si l'on semble initialement dans une rhétorique et une esthétique propres au fantastique, qui tend à mettre en place un cadre mimétique pour ensuite y faire surgir l'inadmissible (Caillois), les enjeux réflexifs de la série confèrent au récepteur un rôle central puisque le fil rouge de Black Mirror est bien de proposer aux spectatrices et spectateurs une confrontation avec des augmentations de l'humain liées au numérique et à la fabrique du vivant. L'altérité renvoie au posthumain (Després/Machinal) et à l'anthropotechnie (Goffette) davantage qu'au surnaturel et à l'ineffable, même si la structure narrative fonctionne bien sur le mode de la rupture, mode propre au fantastique. La défamiliarisation cognitive que proposent à la fois la série et l'épisode s'appuie donc à la fois sur une dialectique entre identité et altérité, humain et posthumain, fiction des possibles et possibles du réel.

The third industrial revolution is currently triggering a redefinition of human identity through evolutions in biotechnologies and screen culture. Television series, especially shows dealing with speculative fiction, reflect the potential evolution and mutation of humanity towards various forms of hybridization, among which the new perspectives opened by the posthuman. This article will focus on "San Junipero", episode S03E04 of the anthology series Black Mirror (Charlie Brooker, Channel 4, Netflix, 2011-), which it will read as a synecdoche for the entire show. The latter indeed rests on a process of defamiliarization often triggered by a novum introduced in the episode - in "San Junipero", the possibility of a virtual eternal life after death. The episode can be analysed as hybrid because it plays on the conventions of both science fiction and the fantastic. It is also highly metareflexive: the viewers play a central part, as the show confronts them virtually, through their screens, to their own posthuman desires: from augmented humanity and digital enhancement to the dystopian mass production of human beings. Through cognitive defamiliarization, the episode under study exemplifies a dialectics of alterity and identity, humanity and posthumanity, fiction and reality.

\section{INDEX}

Keywords : Black Mirror, defamiliarization, hybridity, interface, metafiction, mise en abyme, posthuman, reflexivity, screen, virtual

Mots-clés : Black Mirror, défamiliarisation, écran, hybridité, interface, métafiction, mise en abyme, posthumain, réflexivité, virtuel

\section{AUTEUR}

\section{HÉLÈNE MACHINAL}

Hélène Machinal est professeure à l'Université de Bretagne Occidentale et membre de HCTI (EA 4249). Elle est spécialiste de littérature fantastique, du roman policier et de la fiction spéculative au XIX ${ }^{\mathrm{e}}$ siècle et travaille aussi sur la littérature britannique contemporaine, sur les séries TV, et plus particulièrement les fictions policières, fantastiques et de SF. Ses publications récentes incluent : Le Savant fou, Presses Universitaires de Rennes, 2013 ; PostHumain(s), frontières, évolutions, hybridités (avec Elaine Després), PUR, 2014 ; Mutations I : Corps posthumains (avec JeanFrançois Chassay), Otrante 37, Paris, Kimé, 2015 ; Sherlock (avec Gilles Menegaldo et Jean-Pierre 
Naugrette), PUR, 2016 ; Signatures du monstre (avec Jean-François Chassay et M. MarracheGouraud) PUR, 2017 ; L'imaginaire en séries I (avec Elaine Després), Otrante 42, Paris, Kimé, 2017; et Hybridités posthumaines - cyborgs, mutants, hackers (avec Isabelle Boof-Vermesse et Mathieu Freyheit), Paris, Orizons, 2018.

Hélène Machinal is Full Professor at the University of Bretagne Occidentale, Brest (EA 4249, HCTI). She is a scholar in English Studies, more specifically British literature, from the $19^{\text {th }}$ century to the contemporary period. She focuses on the gothic, science fiction and detective fiction and her recent research is on TV series. Her recent publications include Le Savant fou, Presses Universitaires de Rennes, 2013; PostHumain(s). Frontières, évolutions, hybridités (with Elaine Després), PUR, 2014; Otrante $\mathrm{n}^{\circ}$ 37: Mutations I : Corps posthumains (with Jean-François Chassay), Kimé, 2015; Sherlock (with Gilles Menegaldo \& Jean-Pierre Naugrette) PUR, 2016; Signatures du monstre (with Jean-François Chassay \& M. Marrache-Gouraud) PUR, 2017; L'imaginaire en séries I (with Elaine Després), Otrante 42, Paris, Kimé, 2017, and Hybridités posthumaines - cyborgs, mutants, hackers (with Isabelle Boof-Vermesse and Mathieu Freyheit), Paris, Orizons, 2018. 